CONF-960569--19

GA-A22348

\title{
STABILITY OF A RADIATIVE MANTLE IN ITER
}

\author{
M.A. MAHDAVI, G.M. STAEBLER, R.D. WOOD, \\ D.G. WHYTE and W.P. WEST
}

Mal 17 1

087

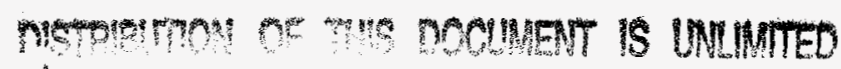

P 


\section{DISCLAIMER}

This report was prepared as an account of work sponsored by an agency of the United States Government. Neither the United States Government nor any agency thereof, nor any of their employees, makes any warranty, express or implied, or assumes any legal liability or responsibility for the accuracy, completeness, or usefulness of any information, apparatus, product, or process disclosed, or represents that its use would not infringe privately owned rights. Reference herein to any specific commercial product, process, or service by trade name, trademark, manufacturer, or otherwise, does not necessarily constitute or imply its endorsement, recommendation, or favoring by the United States Government or any agency thereof. The views and opinions of authors expressed herein do not necessarily state or reflect those of the United States Government or any agency thereof. 


\section{DISCLAIMER}

Portions of this document may be illegible in electronic image products. Images are produced from the best available original document. 


\title{
STABILITY OF A RADIATIVE MANTLE IN ITER
}

\author{
by \\ M.A. MAHDAVI, G.M. STAEBLER, R.D. WOOD, $†$ \\ D.G. WHYTE $¥$ and W.P. WEST
}

This is a preprint of a paper to be presented at the 12th International Conference on Plasma Surface Interactions in Controlled Fusion Devices, May 20-24, 1996, Saint-Raphael, France and to be published in the Proceedings.

$\dagger_{\text {Lawrence Livermore National Laboratory }}$

fINRS - Energie et Materiaux

\section{Work supported by the U.S. Department of Energy \\ under Contract Nos. DE-AC03-89ER51114, and W-7405-ENG-48}




\begin{abstract}
We report results of a study to evaluate the efficacy of various impurities for heat dispersal by a radiative mantle and radiative divertor(including SOL). We have derived a stability criterion for the mantle radiation which favors low $\mathrm{Z}$ impurities and low ratios of edge to core thermal conductivities. Since on the other hand the relative strength of boundary line radiation to core bremsstrahlung favors high $\mathrm{Z}$ impurities, we find that for the ITER physics phase argon is the best gaseous impurity for mantle radiation. For the engineering phase of ITER, more detailed analysis is needed to select between krypton and argon.
\end{abstract}




\section{INTRODUCTION}

One of the most challenging tokamak reactor design problems is exhaust of several hundred megawatts of alpha power $[1,2]$. The heat load problem is greatly aggravated by divertor plate erosion and mechanical and thermal loads due to disruptions. Design constraints imposed by erosion and disruption are in conflict with that of the heat exhaust. For given divertor plasma parameters and target plate material, erosion lifetime and transient disruption loads set a lower bound on the divertor plate thickness whereas the steady state heat load sets an upper limit on the plate thickness. An attractive method of heat dispersal is radiative cooling [2-5]. One important advantage of radiative heat dispersal, as opposed to expansion of the plasma foot print over the target area, is that radiation can virtually eliminate the erosion problem.

In order to radiate the desired fraction of the alpha power, the radiative impurity has to be selected to meet the following criteria: 1-bremsstrahlung and impurity line radiation from the core plasma are to be much less than that of the divertor and the cold plasma boundary, henceforth refered to as the mantle; the impurity emissivity has to be sufficiently high so that the desired level of radiation is achieved at the operating plasma density; and finally the radiative characteristic of the impurity must not lead to an undesirable radiative instability. Any process that enhances impurity radiation in the boundary relative to the core plasma is highly desirable since it relaxes the first two of these constraints. Two such process are divertor impurity enrichment [6] and deviations from Coronal equilibrium caused by finite impurity replacement time or finite neutral density $[11,12,13$ and references thereof].

In the first section of this paper we consider the problem of stability of impurity radiation profiles, where it is found that for the ITER physics phase argon is the best candidate from the point of view of mantle radiation. For lower $Z$ gaseous impurities the ratio of line radiation to bremsstrahlung is too small, whereas for higher $Z$ gaseous impurities the stability boundary limits the radiation to a lower level. In the second half of the paper we address the issue of the feasibility of taking advantage of deviations from coronal equilibrium to improve divertor impurity emissivity. Here we find that the potential gain from deviations from coronal equilibrium cannot be effectively realized in the SOL layer geometry except near the target plate. The problem arises from heat convection along the field lines which must increase as impurity residence time is decreased or neutral density is increased. 


\section{STABILITY OF MANTLE RADIATION}

Several authors [10] have shown that in the absence of divertor impurity enrichment divertor radiation alone is insufficient for heat dispersal; whereas a combination of divertor and core radiation may satisfy the requirements of the physics phase of ITER. Effective mantle radiation has been demonstrated in several existing machines; in particular, in TEXTOR, near $100 \%$ mantle radiation has been obtained with energy confinement of twice ITER89P scaling [7]. In this section we consider the stability of a radiative mantle in the cylindrical geometry .

Here we consider a cylindrical model of the plasma with a reactive core with a constant heating power density $Q$ and a radiative boundary (the mantle) with a radiation power density $R$. It is assumed that the boundary between these two zones is defined by the burnout temperature of the radiating impurity, as shown in Fig. 1. Intuitively, we expect that for a

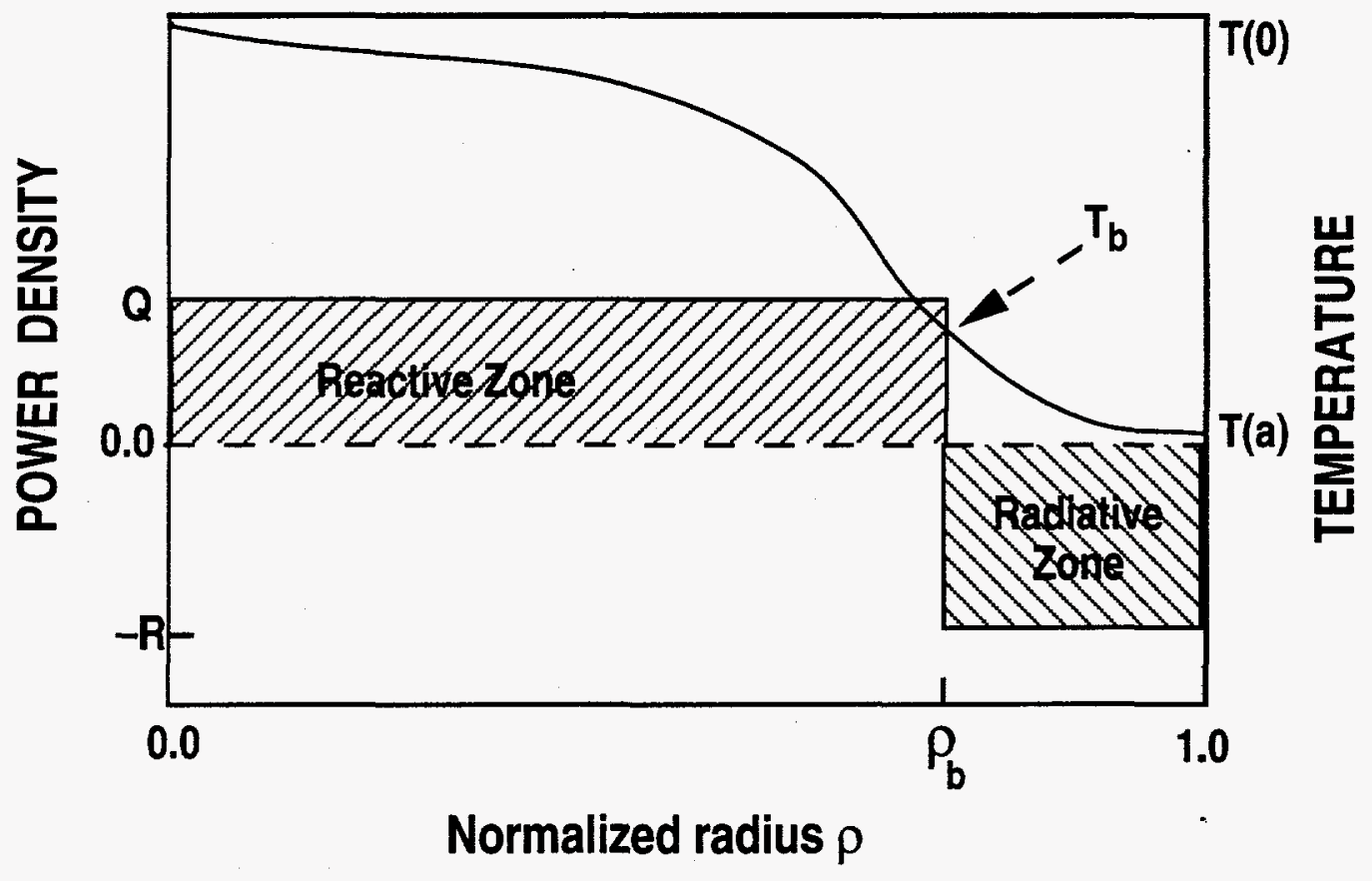

Fig. 1. Model of the core plasma used to calculate the stable radiation fraction. The boundary between the reactive zone and the radiative zone is defined by the burnout temperature of the radiating impurity, $T_{b}$.

given central temperature $T(0)$, as we increase the impurity burnout temperature $T_{b}$, the radius of the boundary shrinks and the total radiated power rises. Thus to maintain the same central temperature requires an increase in the heating power which requires a larger ratio of $Q$ to $R$, and thus a larger discontinuity at the interface of the two zones that can thermally destabilize the plasma. In the following we shall show that the thermal stability of the radiative mantle is 
primarily defined by a single dimensionless parameter $T_{b} T(0) \kappa_{2} / \kappa_{1}$, where $\kappa_{1}$ and $\kappa_{2}$ are thermal conductivities of the core and boundary plasmas, respectively.

In order to find the equilibrium temperature profile, we solve the one dimensional heat transport equation in cylindrical geometry

$$
\begin{aligned}
\frac{1}{\mathrm{r}} \frac{\partial}{\partial \mathrm{r}} \mathrm{r} \frac{\partial}{\partial \mathrm{r}} \kappa \mathrm{T} & =-\mathrm{Q} \mathrm{T} \geq \mathrm{T}_{\mathrm{b}} \\
& =\mathrm{R} \quad \mathrm{T}<\mathrm{T}_{\mathrm{b}}
\end{aligned}
$$

in the two regions of interest with the boundary conditions of matching temperatures and heat fluxs at the interface where $T=T_{b}$. Defining the following dimensionless parameters

$$
\rho=\frac{\mathrm{r}}{\mathrm{a}}, \theta=\frac{\kappa_{1} \mathrm{~T}}{\mathrm{a}^{2} \mathrm{Q}}, \mu=\frac{\kappa_{2}}{\kappa_{1}} \text {, and } \gamma=\frac{\mathrm{R}}{\mathrm{Q}} \text {, }
$$

where $\theta$ and $\rho$ are the normalized temperature and radius, we obtain

$$
\begin{aligned}
\frac{1}{\rho} \frac{\partial}{\partial \rho} \rho \frac{\partial \theta}{\partial \rho} & =-1 \quad \theta \geq \theta_{\mathrm{b}} \\
& =\frac{\gamma}{\mu} \quad \theta<\theta_{\mathrm{b}}
\end{aligned}
$$

with boundary conditions:

$$
\begin{array}{ll}
\left.\frac{\partial \theta}{\partial \rho}\right|_{0}=0 & \left.\frac{\partial \theta}{\partial \rho}\right|_{\rho_{\mathrm{b}}}=\left.\mu \frac{\partial \theta}{\partial \rho}\right|_{\rho_{\mathrm{b}}+\varepsilon} \\
\left.\theta\right|_{1}=\theta_{\mathrm{s}} & \left.\theta\right|_{\rho_{\mathrm{b}}}=\left.\theta\right|_{\rho_{\mathrm{b}}+\varepsilon}
\end{array}
$$

Here $\rho_{b}$ is radius of the mantle and $\theta_{\mathrm{s}}$ is the separatrix temperature. Integrating Eq. (1) and applying the boundary conditions of Eq. (3), we obtain

$$
\begin{aligned}
\theta & =\theta_{0}-\frac{\rho^{2}}{4} \text { for } \rho \leq \rho_{\mathrm{b}} \\
& =\frac{1}{\mu}\left\{\gamma \frac{\rho^{2}}{4}-\frac{\rho_{\mathrm{b}}^{2}}{2}(1+\gamma) \ln \rho-\frac{\gamma}{4}\right\}+\theta_{\mathrm{s}}, \text { for } \rho>\rho_{\mathrm{b}}
\end{aligned}
$$


where

$$
\theta_{0}=\frac{\rho_{\mathrm{b}}^{2}}{4}+\frac{1}{\mu}\left\{\frac{\gamma \rho_{\mathrm{b}}^{2}}{4}-\frac{\rho_{\mathrm{b}}^{2}}{2}(1+\gamma) \ln \rho_{\mathrm{b}}-\frac{\gamma}{4}\right\}+\theta_{\mathrm{s}}
$$

and $\rho_{b}$ is to be determined from

$$
\theta_{\mathrm{b}}=\frac{1}{\mu}\left\{\gamma \frac{\rho_{\mathrm{b}}^{2}}{4}-\frac{\rho_{\mathrm{b}}^{2}}{2}(1+\gamma) \ln \rho_{\mathrm{b}}-\frac{\gamma}{4}\right\}+\theta_{\mathrm{s}}
$$

Up to this point, our approach is similar to that of Drake [8], who used a constant heating function and thermal conductivity accross the whole plasma, and identical to that of Stacy [9]. Here we deviate from the previous authors by deriving the stability condition in the exact cylindrical geometry. Performing a local stability analysis similar to that in Drake [8] we obtain the stability requirement.

$$
1+2 \ln \left(\rho_{b}\right)(1+\gamma) \geq 0
$$

This same condition follows from solving Eq. (5) for $Q\left(\rho_{b}\right)$ and requiring that $d Q / d \rho_{b} \geq$ 0 . This is physically plausible since it requires that the edge radiation zone shrink in size when the heating power increases. Using the equal sign in Eq. (7), we fined the minimum stable value of $\rho \equiv \rho_{c}$.

$$
\rho_{\mathrm{c}}=\exp \left[-\frac{1}{2(1+\gamma)}\right]
$$

If the equilibrium value of $\rho_{b}$ is less than $\rho c$, then a small drop in heating power density $Q$ would result in collapse of the core plasma.

It is straitforward to compute the critical radiation fraction beyond which the plasma is thermally unstable.

$$
F_{c}=\gamma\left(e^{\frac{1}{1+\gamma}}-1\right)
$$

Using the stability limit of $\gamma$ given by expression (7), in Eqs. (5) and (6), we find

$$
\xi \equiv \mu\left(\theta_{\mathrm{b}}-\theta_{\mathrm{s}}\right) /\left(\theta_{0}-\theta_{\mathrm{b}}\right)=\left(\frac{1+2 \ln \rho_{\mathrm{c}}}{2 \ln \rho_{\mathrm{c}}}\right)\left(1-2 \ln \rho_{\mathrm{c}}-\rho_{\mathrm{c}}^{-2}\right)-2 \ln \rho_{\mathrm{c}}
$$


Equation (10) shows that $\rho_{c}$ is only a function of the dimenssinless parameter $\xi=$ $\left(T_{b}-T(a) / T(0)-T_{b}\right) \kappa_{2} / \kappa_{1}$ and consequently the maximum allowed radiation fraction $F_{c}$ is also only a function of $\xi$. In Fig. 2 we have plotted $\rho_{c}$ and the maximum radiated radiation fraction versus $\xi$. This figure shows that the allowed radiation fraction decreases with increasing $T_{b} / T(0)$ and increasing ratio of the edge to core thermal conductivity $\kappa_{2} / \kappa_{1}$. Thus stability favors low $Z$ impurities and confinement modes with low values of $\kappa_{2} / \kappa_{1}$.

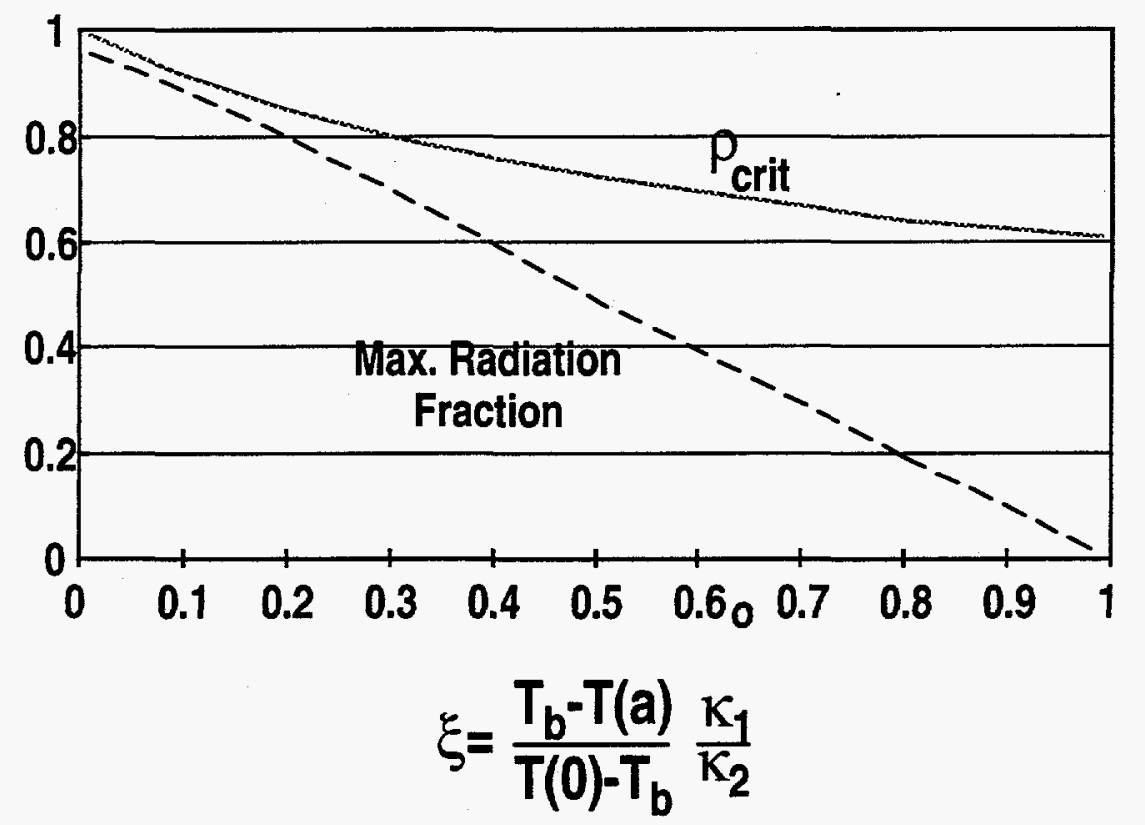

Fig. 2. The maximum allowed radiation fraction and the critical radius are plotted against parameter $\xi$ which characterizes the mantle stability. Maximum radiation fraction decreases with increasing impurity burnout temperature and ratios of edge to core conductivies.

Assuming $\mu=1, \mathrm{~T}_{\mathrm{s}}=0, \mathrm{~T}_{0}=22 \mathrm{keV}$, and $\mathrm{T}_{\mathrm{b}} \simeq 7 \times \mathrm{XZ}^{2} \mathrm{eV}$ we find that for argon and lower $\mathrm{Z}$ impurities $\geq 90 \%$ radiated fraction is allowable; whereas for krypton it is $-30 \%$.

Although, stability favors low $\mathrm{Z}$ impurities, requiring low bremsstrahlung radiation in the reactive core favors high $\mathrm{Z}$ impurities. Using the approximate formulas

$$
\mathrm{R}_{\mathrm{CE}} \simeq 10^{-35} \mathrm{Z}^{2} \mathrm{n}_{\mathrm{e}} \mathrm{n}_{\mathrm{i}} \int_{\rho_{\mathrm{b}}}^{1} \rho \mathrm{d} \rho\left(\mathrm{Wm}^{-3}\right)
$$

and

$$
\mathrm{R}_{\mathrm{Brem}} \cong 4.8 \times 10^{-37} \mathrm{Z}^{2} \mathrm{n}_{\mathrm{e}} \mathrm{n}_{\mathrm{i}} \int_{0}^{\rho_{\mathrm{b}}} \sqrt{\mathrm{T}(\mathrm{keV})} \rho \mathrm{d} \rho\left(\mathrm{Wm}^{-3}\right)
$$


for coronal equilibrium and bremsstrahlung, respectively, and using the results of Eq. (3), we obtain

$$
\begin{aligned}
& \frac{\mathrm{R}_{\mathrm{ce}}}{\mathrm{R}_{\mathrm{Brem}}} \simeq \frac{32 \mu}{\sqrt{\mathrm{T}_{0}}}\left(\frac{\frac{\mathrm{T}_{\mathrm{b}}}{\mathrm{T}_{0}}}{1-\frac{\mathrm{T}_{\mathrm{b}}}{\mathrm{T}_{0}}}\right) \frac{\left[1-2 \mu\left(\frac{\mathrm{T}_{\mathrm{b}} / \mathrm{T}_{0}}{1-\frac{\mathrm{T}_{\mathrm{b}}}{\mathrm{T}_{0}}}\right)\right]^{1 / 2}}{\left(1-\frac{\mathrm{T}_{\mathrm{b}}}{\mathrm{T}_{0}}\right)^{1 / 2}\left[1-\left(\frac{\mathrm{T}_{\mathrm{b}}}{\mathrm{T}_{0}}\right)^{3 / 2}\right]}, \\
& \frac{\mathrm{R}_{\mathrm{ce}}}{\mathrm{R}_{\mathrm{Brem}}} \approx \frac{32}{\sqrt{\mathrm{T}_{0}(\mathrm{keV})}} \mu \frac{\mathrm{T}_{\mathrm{b}}}{\mathrm{T}_{0}}\left(1-\mu \frac{\mathrm{T}_{\mathrm{b}}}{\mathrm{T}_{0}}\right),
\end{aligned}
$$

where we have approximated $\theta_{0}$ by $1 / 4$ and ignored terms of the order of $T_{s} / T_{b}$ and $T_{b} / T_{0}$. Assuming $T_{0}=22 \mathrm{keV}$ and $\mu=1$, then the ratio $R_{C E} / R_{B r e m}$ is $0.2,0.9$ and 7 for neon, argon, and krypton respectively. Thus the undesirable bremsstrahlung from the core exceeds the mantle radiation for neon and lighter(i.e. lower $\left.\mathrm{T}_{b}\right)$ impurities. 


\section{DIVERTOR RADIATION AND DEVIATIONS FROM CORONAL EQUILIBRIUM}

Estimates of divertor (here by divertor we imply the entire open field line plasma volume) radiation using the coronal equilibrium radiation model Post [10] showed that argon and neon are equally the best divertor radiators. Using Post's results, and the results of the above core model, we conclude that overall argon is the best candidate impurity for ITER. It is well known that charge exchange collisions and finite impurity residence time can cause large deviations from coronal equilibrium $[11,12]$. Both effects can increase impurity emissivities (to an enhanced value $L^{\prime}$ ) by several orders of magnitude. It has been proposed [13] that it may be possible to take advantage of the deviations from coronal equilibrium to enhance divertor radiation. Here we will show that in the divertor it is not feasible to significantly reduce the divertor heat load on the basis of deviations from coronal equilibrium, except perhaps in a shallow region near the divertor target plate, and unless the impurity perpendicular transport greatly exceeds that of the working gas. We shall shortly show that under steady state conditions, to take advantage of either of these effects requires a selfconsistent flow along the field lines. The magnitude of this flow increases towards the plate such that the increase in convected heat along the field lines for neon and higer $\mathrm{Z}$ impurities is always much larger than the enhanced radiative dissipation, and thus inconsistent with the goal of reducing the heat flux to the target plate.

Using the geometry shown in Fig. 3, we note that finite neutral density leads to an ionization source $S=n_{e} n_{0}<\sigma_{i o n} v>$. The continuity and energy conservation equations are

$$
\begin{aligned}
& \nabla \cdot(\mathrm{nV})=\mathrm{S} \\
& \nabla \cdot\left(\mathrm{q}_{\mathrm{s}}+5 \mathrm{nVT}\right)=-\mathrm{n}_{\mathrm{i}} \mathrm{n}_{\mathrm{e}} \mathrm{L}^{\prime}\left(\mathrm{T}, \frac{\mathrm{n}_{0}}{\mathrm{n}_{\mathrm{e}}}\right)
\end{aligned}
$$

where $q_{\|}$is the conduction heat flux along the field lines, $L^{\prime}$ is the enhanced emissivity, and $\mathrm{n}_{0}$ is the neutral density. Integrating these equations over the desired segment of the SOL with enhanced emissivity from upstream point (1) to downstream point (2), and requiring that at least $3 / 4$ of the initial heat flowing into the integration volume is radiated i.e., $\int_{1}^{2} \mathrm{n}_{\mathrm{j}} \mathrm{n}_{\mathrm{e}} \mathrm{L}^{\prime} \mathrm{d} l=\frac{3}{4} \mathrm{q}_{\|}(1)>\mathrm{q}_{\|}(2)$, we obtain 


$$
\mathrm{n}_{2} \mathrm{~V}_{2} \cong l \mathrm{n}_{\mathrm{e}} \mathrm{n}_{0}<\sigma_{\text {ion }} \mathrm{v}>\approx\left(\frac{\mathrm{n}_{0}}{\mathrm{n}_{\mathrm{e}}}\right)<\sigma \mathrm{v}>\int_{1}^{2} \mathrm{n}_{\mathrm{e}}^{2} \mathrm{~d} \ell
$$

$$
\frac{Z_{\text {eff }-1}}{Z^{2}} \int_{1}^{2} n_{\mathrm{e}}^{2} L^{\prime} \mathrm{d} l \cong 15 \mathrm{~T}_{2} \mathrm{n}_{2} \mathrm{~V}_{2}+3 \mathrm{q}_{\|}(2)
$$

$$
\begin{aligned}
& \text { Assuming } \int_{1}^{2} \mathrm{n}_{\mathrm{e}}^{2} \mathrm{~L}^{\prime} \mathrm{d} l \leq \mathrm{L}_{2}^{\prime} \int_{1}^{2} \mathrm{n}_{\mathrm{e}}^{2} \mathrm{~d} \ell, \\
& \frac{\mathrm{n}_{\mathrm{e}}}{\mathrm{n}_{0}} \mathrm{~L}^{\prime}\left(\mathrm{T}_{2}, \frac{\mathrm{n}_{0}}{\mathrm{n}_{\mathrm{e}}}\right) \geq 15 \frac{\mathrm{Z}^{2}}{\mathrm{Z}_{\mathrm{eff}-1}}<\sigma_{\text {ion }} \mathrm{v}>\mathrm{T}_{2} .
\end{aligned}
$$

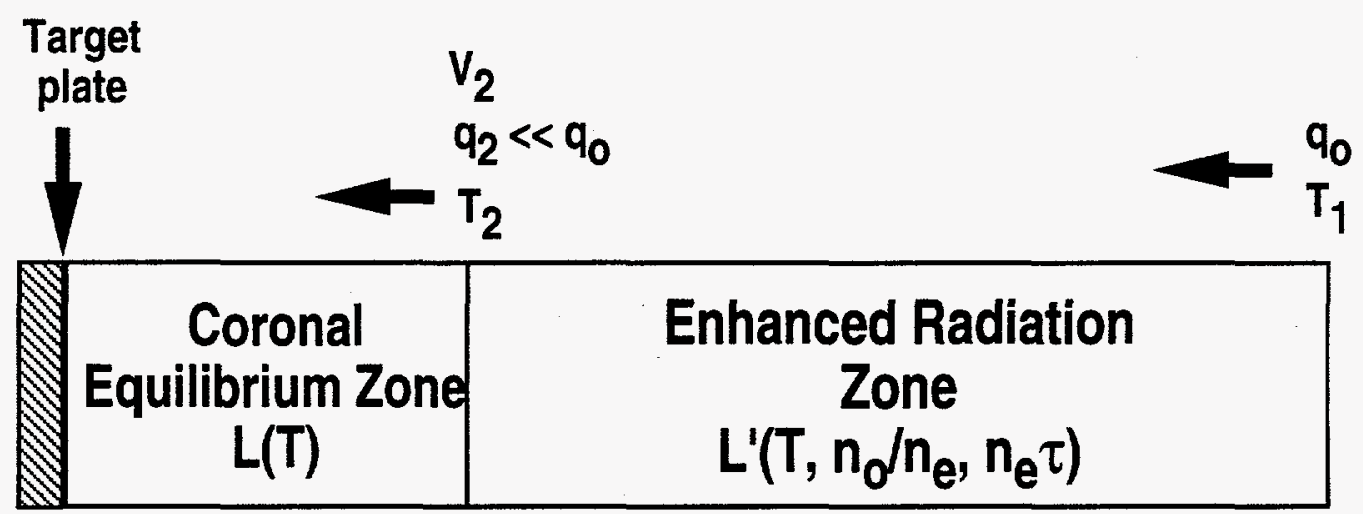

Fig. 3. Model of the SOL used to estimate the convected heat flux resulting from a finite neutral density. Neutrals are assumed to produce a uniform neutral density in the SOL

We have selected the end point such that $T_{2}$ is the temperature below which $L^{\prime}$ is not significantly above the coronal equilibrium value. This result shows that $n_{0} / n_{e}$ cannot be arbitarily increased unless $L^{\prime}$ increases at least as fast as $n_{0} / n_{e}$. Noting that $T_{2} \approx 40 \mathrm{eV}$ for neon, and using $\mathrm{n}_{0} / \mathrm{n}_{\mathrm{e}}=10^{-3}, \mathrm{~L}^{\prime}\left(40 \mathrm{eV}, 10^{-3}\right) \leq 10^{-25} \mathrm{Wcm}^{3}, \mathrm{Z}_{\mathrm{eff}}=1.5$, and $\langle\sigma \mathrm{V}\rangle \geq 4 \times 10^{-8}$ $\mathrm{cm}^{3} / \mathrm{s}^{-1}$, it is found that the right hand side is 8 times larger than the left hand side, thus the inequality can not be satisfied, therefore violating the assumption that $3 / 4$ of the power is radiated.

We reach a similar conclusion for the case of the finite impurity life time. In this case a flow is required since impurities must be recycled at the divertor plate. Assuming that the impurities are introduced uniformly in the SOL as neutrals and after ionization flow to the 
plate at the background plasma speed, and following the same procedures as above we obtain.

$$
\mathrm{n}_{\mathrm{e}} \tau \mathrm{L}^{\prime}\left(\mathrm{T}_{2}, \mathrm{n}_{\mathrm{e}} \tau\right) \geq \frac{15 \mathrm{Z}^{2}}{\mathrm{Z}_{\mathrm{eff}-1}} \mathrm{~T}_{2}
$$

In this case for neon using $n_{e} \tau=10^{11} \mathrm{~cm}^{-3} \mathrm{~s}, \mathrm{~L}^{\prime}$ is in the range of $1-10 \times 10^{-26} \mathrm{Wcm}^{3}$, we find that the right hand side is twice the left hand side thus the inequality can not be satified, again violating the requirement that $75 \%$ intial heat flux is dissipated by the enhanced coronal radiation. Conditions 17 and 18 are easier to satisfy for lighter elements because of smaller $Z^{2}$ and $T_{2}$ values on the right hand side of both equations. For nitrogen, the lowest $Z$ gaseous impurity, condition 18 can be satisfied. However, most of the gain is in the region of $10-30 \mathrm{eV}$, near the divertor plate, and at best the increased emissivity would make nitrogen performance comparable to neon only in the divertor. The result here does not necessarily apply to either perpendicular transport of impuritites or a case with counter flows along the field lines. In such cases, the relationship between the convective heat loss and finite impurity residence time is broken. Finally it should be emphasized that the above results do not preclude the possibility of impurity radiation locally far exceeding the coronal equilibrium level.

Since in coronal equilibrium argon and neon are roughly the best divertor radiators for ITER [10] this conclusion is not significantly changed by deviations from coronal equilibrium. We conclude that overall argon is the best radiator for the physics phase of ITER, since in the core plasma argon is a more desirable radiator. 


\section{SUMMARY AND DISCUSSION}

In summary, we find that the fraction of power that can be radiated in the plasma boundary of ITER is limited by the the thermal stability of the boundary between the reactive core and the radiative mantle. A stability criterion is derived that favors low $\mathrm{Z}$ impurities and low ratios of edge to core thermal conductivities. Since on the other hand bremsstrahlung and fuel dilution in the core favors high $\mathrm{Z}$ impurities, we find that overall argon is the best gaseous impurity for mantle radiation for the ITER physics phase. In the SOL, it is shown it is not feasible to take advantage of deviations from coronal equilibrium to significantly reduce the divertor heat load. Thus using the results of Post that argon and neon are the two best SOL radiators and roughly equal, we concluded that argon is the best overall radiator for the physics phase of ITER.

Our mantle model exaggerates the instability because of the sharp boundary of the radiation and reactive zones. On the other hand, since it assumes a temperature independent heating source inside $\rho=\rho_{b}$, it tends to understate the instability. Thus, although, our rough analysis shows that argon is better than krypton for edge cooling, improved analysis could change this relationship, paticularly if a higher central temperature is allowed, as is the case for the engineering phase of ITER. Therefore it is important to develop a time dependent numerical model of the core plasma.

Furthermore, we assumed the ratio of edge to core thermal conductivities to be 1 . Although this assumption is consistent with DIII-D ELM-free $\mathrm{H}$-mode data for $\rho<0.8$, it may not be justifiable for larger $\rho$ values or other confinement modes. We also note, that had we allowed the heating profile to be constant across the whole plasma profile, it would have resulted in the stability condition $\gamma<-1 / 2 \ln \rho_{c}$, which is less restrictive than the equilibrium condition $\gamma\left(\frac{1-\rho_{\mathrm{c}}^{2}}{\rho_{\mathrm{c}}^{2}}\right) \leq 1$. Thus profile stability results from present machines with auxiliary heating do not necessarily apply to burning plasmas. 


\section{REFERENCES}

[1] G. Janeschitz, et al., Journ. of Nucl. Mater. 220-222 (1995) 73.

[2] D.E. Post, et al., ITER Doc. Series No. 21 IAEA, Vienna, Austria (1991).

[3] N. Ohyabu, Nucl. Fusion 9 (1979) 1491.

[4] M. Shimada, et al., Nucl. Fusion 22 (1982) 643.

[5] G.C. Vlases, Plasma Phys. 35 (1993) B67.

[6] Schaffer, these proceedings.

[7] U. Samm, Trans. Fusion Technol. 25259 (1994).

[8] J.F. Drake, Phys. Fluids 30 (8) (1987) 2429.

[9] W.M. Stacy, Phys. Fluids 3 (3) (1996) 1012.

[10] D. Post, N. Putvinskaya, F.W. Perkins, W. Nevins, Journ. Nucl. Mater. 220-222 (1995) 1014.

[11] P.G. Carolan and V.A. Piotrowicz, Plasma Physics, Vol. 25, No. 10, (1983) 1065.

[12] S.L. Allen, et al., J. Nucl. Mater. 196-198 (1992) 804.

[13] R.A. Hulse, D.E. Post, and D.R. Mikkelson, J. Phys. B: Atom. Molec. Phys. 13 (1980) 3895-3907. 


\section{ACKNOWLEDGMENT}

Work supported by the U.S. Department of Energy under Contract Nos. DE-AC0389ER51114 and W-7405-ENG-48. 